Recepción: 19/05/2018

Aceptación: 27/06/2019

Publicación: 05/08/2019

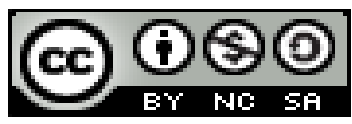

Ciencias de la Educación

Artículo de investigación

\title{
Elementos gerenciales en la productividad académica del docente universitario
}

Management elements in the academic productivity of the university teacher

\section{Elementos gerenciais na produtividade acadêmica do professor universitário}

\author{
Carlos Enrique Santos-Loor $\stackrel{I}{-}$ \\ carlossantosloor@hotmail.com \\ Carlina Patricia Santos-Loor II \\ pasalo1966@hotmail.com
}

Hugo Jesús Juan Vélez-Pincay ${ }^{\text {III }}$

hvelez99@hotmail.com

Christian Alfredo Cevallos-Arteaga ${ }^{\text {IV }}$

cristhicevall@hotmail.com

Maria Vanesa Zamora-Lucas $v$

vane_zamora_lucas@hotmail.es

Correspondencia: carlossantosloor@hotmail.com

I. Doctor en Ciencias de la Educación Especialización Historia y Geografía, Magíster en Docencia e Investigación Educativa, Licenciado en Ciencias de la Educación Especialización Historia y Geografía, Docente de la Universidad Técnica de Manabí, Portoviejo, Ecuador.

II. Doctora en Ciencias de la Educación Mención Investigación Educativa, Magíster en Desarrollo Educativo, Licenciada en Ciencias de la Educación Especialidad Psicología y Orientación Vocacional, Docente de la Universidad Técnica de Manabí, Portoviejo, Ecuador.

III. Magíster en Educación y Desarrollo Social, Licenciado en Ciencias de la Educación Especialización Química y Biología, Docente de la Universidad Técnica de Manabí, Portoviejo, Ecuador.

rv. Maitrise Universitaire en Sciences de L'education - Analyse et Intervention dans les Systemes Educatifs, Baccellierato in Teologia, Docente de la Universidad Técnica de Manabí, Portoviejo, Ecuador.

v. Licenciada en Ciencias de la Educación Mención Educación Primaria, Docente Unidad Educativa Fiscal Franklin Delano Roosevelt, Portoviejo, Ecuador. 


\section{Resumen}

Esta investigación pretende diseñar un modelo de gerencia estratégica para el fomento de la productividad científica del profesor universitario desde una perspectiva del materialismodialéctico en la Universidad. El trabajo está basado en los supuestos gnosiológicos de Sallenave (2002), Fonden (2005), Jones y George (2006), David (1999), Frasi (2007), y Jiménez 81994), entre otros. Desde el punto de vista paradigmático se sustenta en el modelo del materialismo histórico según Toro y Hurtado (2010), desde la perspectiva del materialismo-dialéctico según Castellano Simons (2005), Cerezal y Fiallo (2004), en el contexto del cual se aplicarán métodos teórico-empíricos y estadísticos, asimismo se emplearán métodos como el análisis de contenido, el analítico-sintético, el histórico-lógico, inductivo, deductivo, la observación y la entrevista, según Barreras (2009), para la recolección de la data de una muestra teórica de 05 profesores universitarios que ostentan cargos de gerencia en la referida institución de Educación Superior y 04 profesores. El objeto de investigación es la Gerencia en la educación Universitaria y la Producción científica; y el campo de acción es la gerencia estratégica en los procesos de productividad científica del profesor universitario. Se presentará el modelo de gerencia estratégica siguiendo los postulados y componentes propuestos por Valle Lima (2010). Entre las consideraciones finales que se presentan en esta versión preliminar del proyecto destacan las que siguen a continuación: se precisa la implementación de un modelo educativo de gerencia estratégica a tenor de fomentar la productividad científica en franca y directa relación con los tres procesos sustantivos de la función del docente universitario: docencia, investigación y extensión.

Palabras Clave: Gerencia estratégica; productividad académica; profesor universitario

\section{Abstract}

This research aims to design a model of strategic management for the promotion of the scientific productivity of the university professor from a perspective of dialectical materialism in the University. The work is based on the gnosiological assumptions of Sallenave (2002), Fonden (2005), Jones and George (2006), David (1999), Frasi (2007), and Jiménez 81994), among others. From the paradigmatic point of view, it is based on the model of historical materialism according to Toro y Hurtado (2010), from the perspective of dialectical materialism according to 
Castellano Simons (2005), Cerezal and Fiallo (2004), in the context of which they will apply Theoretical-empirical and statistical methods will also employ methods such as content analysis, analytical-synthetic, historical-logical, inductive, deductive, observation and interview, according to Barreras (2009), for the collection of data from a theoretical sample of 05 university professors holding management positions in the aforementioned institution of Higher Education and 04 professors. The object of investigation is the Management in the University education and the Scientific production; and the field of action is strategic management in the processes of scientific productivity of the university professor. The strategic management model will be presented following the postulates and components proposed by Valle Lima (2010). Among the final considerations presented in this preliminary version of the project are those that follow: the implementation of an educational model of strategic management is required in order to promote scientific productivity in direct and frank relation with the three substantive processes of the role of the university teacher: teaching, research and extension.

Keywords: Strategic management; academic productivity; college professor

\section{Resumo}

Esta pesquisa tem como objetivo projetar um modelo de gestão estratégica para a promoção da produtividade científica do professor universitário a partir de uma perspectiva do materialismo dialético na universidade. O trabalho é baseado nas premissas gnosiológicos Sallenave (2002), Fonden (2005), Jones e George (2006), David (1999), Frasi (2007) e Jimenez 81994), entre outros. De um ponto de vista paradigmático é baseado no modelo do materialismo histórico como Toro y Hurtado (2010), a partir da perspectiva do materialismo-dialético de acordo castelhanos Simons (2005), Cerezal e Fiallo (2004), no contexto da qual é aplicável métodos empíricos e teóricos estatísticos também métodos como análise de conteúdo, o analítico-sintético, o histórico e lógico, indutivo, dedutivo, observação e entrevista ser utilizados como barreiras (2009), para recolher os dados de uma amostra teórica de 05 professores universitários titulares de cargos de gestão na referida instituição de ensino superior e 04 docentes. O objeto de investigação é a Gestão na educação universitária e a produção científica; e o campo de ação é a gestão estratégica nos processos de produtividade científica do professor universitário. O modelo de gestão estratégica será apresentado seguindo os postulados e componentes propostos por Valle Lima 
(2010). Entre as considerações finais apresentadas nesta versão preliminar da proposta de destaques que se seguem: a implementação de um modelo educacional do abrigo gestão estratégica para promover a produtividade científica em relação franca e direta com os três processos substantivos necessários papel do professor universitário: ensino, pesquisa e extensão.

Palavras-chave: Gestão estratégica; produtividade acadêmica; professor universitario

\section{Introducción}

Hoy en día la educación está signada por cambios que han venido evolucionando con el paso del tiempo, ello implica la capacidad de adaptación para ofrecer respuestas a las problemáticas que se suscitan en la actualidad. Asimismo, este vertiginoso mundo contextual marcado por las transformaciones implica en sí mismo la ingente necesidad de proporcionar producción de conocimientos que coadyuven en la solucionática de las situaciones dilemáticas que enfrenta hoy la denominada sociedad del conocimiento y de la información. Ello se configura en el mundo universitario, en la necesidad de conformarse en una institución que proporcione conocimientos y producción científica en correspondencia con las exigencias competitivas de la sociedad actual.

La institución universitaria por tanto, debe responder ante tantos retos y desafíos que plantea el siglo XXI en el mundo y en América Latina en particular, porque estos cambios y estas transformaciones inciden y se relacionan directamente con el ámbito científico-tecnológico de las instituciones educativas y de las universitarias en especial, influyendo a su vez en el trazado de sus objetivos organizacionales y de desarrollo en aras de elevar la calidad educativa que proporciona, de modo que propenda a elevar la calidad de vida de los ciudadanos en general.

Tradicionalmente, las instituciones universitarias forman profesionales de calidad en diversos ámbitos del saber, esta formación privilegia el ámbito de las ciencias sociales y por ende responde a los requerimientos del contexto en el que se imbrica, ello comporta en sí mismo el hecho de que en el cumplimiento y desarrollo del rol de la institución universitaria en sociedad amerite de una gestión que permita el fomento y la ejecución de la productividad del personal académico universitario. Debe ser una gestión que se destaque por el uso de las estrategias técnicas con eficiencia y eficacia en el alcance de metas y proyectos previamente propuestos en su planificación.

\section{4}

Pol. Con. (Edición núm. 36) Vol. 4, No 8, agosto 2019, pp. 71-94, ISSN: 2550 - 682X 
De allí que las universidades se conciben como centros de productividad intelectual, (mediante la ejercitación de las estrategias de las tres funciones universitarias sustantivas: docencia, investigación y extensión, que genera conocimientos, tecnología, innovación y soluciones a los problemas planteados), estos tres procesos deben desarrollarse en forma conjunta integrada e integradora.

Esta investigación pretende diseñar un modelo de gerencia estratégica para el fomento de la productividad científica del profesor universitario desde una perspectiva del materialismodialéctico en la Universidad Técnica de Manabí. El trabajo está basado en los supuestos gnosiológicos de Sallenave (2002), Fonden (2005), Jones y George (2006), David (1999), Frasi (2007), y Jiménez 81994), entre otros. Desde el punto de vista paradigmático se sustenta en el modelo del materialismo histórico según Toro y Hurtado (2010), desde la perspectiva del materialismo-dialéctico según Castellano Simons (2005), Cerezal y Fiallo (2004), en el contexto del cual se aplicarán métodos teórico-empíricos y estadísticos tales como el análisis de contenido, el analítico-sintético, el histórico-lógico, inductivo, deductivo, la observación y la entrevista, Barreras (2009), entre otros para la recolección de la data a una muestra teórica de 05 profesores universitarios que ostenten cargos gerenciales en la referida institución de Educación Superior. El objeto de investigación es la Gerencia en la educación Universitaria y la Producción científica y el campo de acción es la gerencia estratégica en los procesos de productividad científica del profesor universitario de la universidad Técnica de Manabí

\section{Materiales y métodos}

\section{Idea impulsadora de la investigación}

Desde la experiencia empírica del investigador se ha podido observar en la Universidad Técnica de Manabí que la docencia, la investigación y la extensión han sido vistas y desarrolladas en forma fragmentaria, parcelada, atomista, las investigaciones se realizan solo para cumplir requisitos de ascenso en el escalafón del profesor universitario, las que se logran no se publican con la frecuencia requerida, el alcance de patentes es poco significativo, no se concretan redes de investigadores, en razón de líneas de investigación; aunado a lo anterior, el profesor universitario no integra las tres funciones sustantivas y se limita en su productividad científica porque no se estimula, y no se subvenciona suficientemente. Tal situación obedece a una serie de elementos 
multifactoriales entre los que destaca la alta gerencia (decanos, vicerrectores), la gerencia media (directores de programa y jefes de departamento), la gerencia baja (coordinadores de unidades y centros de investigación), cuyos involucrados son los llamados a impulsar los cambios necesarios que permitan el fomento de la productividad científica de los profesores universitarios de la referida Universidad Técnica de Manabí

Desde este contexto, se asume que el enfoque filosófico-epistémico sobre el tema de la gerencia estratégica para el fomento de la productividad científica reviste una relevancia significativa, en el entendido de que se trata de la gestión para la producción de conocimientos, que se asume desde una posición transdisciplinar en pos de dar respuestas a los problemas actuales de la sociedad; en correspondencia con la postmodernidad, y en correspondencia con nuevas maneras de comprender la realidad compleja en la que se vive hoy en día. Las universidades están comprometidas en este reto, el cual ha sido suscrito desde la Cumbre Mundial de la Educación Superior celebrada en París (1998).

Desde ese marco contextual, América Latina se circunscribe ante la necesidad de implementar innovaciones y cambios de la Educación Universitaria. En ese sentido, en Ecuador se abren nuevas perspectivas hacia la renovación de la institución universitaria y el consecuente impulso que ello implica en los procesos de gerencia para el fomento de la productividad científica del profesor universitario. A la luz de estas premisas se presentan los supuestos gnoseológicos esenciales que sustentan esta investigación de la siguiente guisa:

\section{Supuestos gnoseológicos esenciales sobre modelo educativo}

La modelación como método de conocimiento científico ha venido desarrollándose, a partir de formas muy simples hasta los recientes métodos teóricos del conocimiento científico. A los fines de este trabajo es útil definirla para entender su especificidad y relacionarla con los aspectos que involucran el diseño de un modelo educativo.

En ese sentido, es conveniente aclarar que: La modelación es un método científico que opera de forma práctica y teórica con un objeto no en forma directa sino utilizando cierto sistema intermedio natural o artificial el cual se encuentra en una determinada correspondencia objetiva con el objeto mismo del conocimiento (Bergues, s.f). Esta autora apunta que la modelación

\section{6}

Pol. Con. (Edición núm. 36) Vol. 4, No 8, agosto 2019, pp. 71-94, ISSN: 2550 - 682X 
científica es un método por el cual se ejecuta de manera teórica y práctica un sistema intermedio natural o artificial (modelo) a partir del objeto de estudio, con el propósito de analizar la realidad y ofrecer mejoras a la situación presentada.

A la luz de estas consideraciones se adopta la concepción del Centro de Asesoría Pedagógica (s.f), donde se establece que los modelos educativos son visiones sintéticas de teorías o enfoques pedagógicos que orientan a los especialistas y a los profesores en la elaboración y análisis de los programas de estudios; en la sistematización del proceso de enseñanza-aprendizaje, o bien en la comprensión de alguna parte de un programa de estudios. Visto desde esta perspectiva, los modelos educativos son estándares conceptuales que ayudan a esquematizar las partes y los elementos de un programa de estudio y a simplificar el quehacer educativo. Tomando como referencia estas definiciones, el investigador coincide con Chirino y Lamus (2016), quienes sostienen que un modelo educativo es una compilación de teorías y enfoques pedagógicos que guían a los expertos en el área educativa a perfilar las partes y los elementos de un programa de estudio, a comprender alguna parte del mismo o a simplificar el quehacer educativo.

Desde este marco referencial, según los autores citados, en el mundo existen modelos educativos que responden a demandas y retos del siglo XXI. En el Sistema Educativo Ecuatoriano se corresponde con la Constitución, con la Ley Orgánica de Educación Universitaria, en cuyo contexto, la Universidad Técnica de Manabí consciente de su compromiso social en el proceso de generación y gestión de conocimientos debe atender y coadyuvar hacia el fortalecimiento de la productividad académica del profesor universitario.

En ese orden, cabe considerar, en primer lugar, cuáles son los componentes que rigen la elaboración de un modelo educativo. En ese sentido, se observó en la revisión de la literatura referida a los componentes de un modelo educativo que existen discrepancias entre los autores consultados. Así, Jara (2008) sostiene que los aspectos a tomar en cuenta en un modelo educativo vendrían a ser: el marco filosófico, donde se describe el concepto de hombre, las finalidades de educación, el concepto de sociedad, y en este caso el modelo de gerente estratega para el fomento de la productividad científica del profesor universitario y el marco legal, en donde se reflejan las leyes que legitiman el modelo; el marco organizativo, las modalidades académicas los mecanismos de control, entre otros. 
Desde la concepción de la autora citada, un modelo educativo debe contener una serie de componentes representados por marcos de diferentes tipos; los cuales deben proporcionar una visión general a los docentes para realizar la planeación institucional. Por su parte, Pérez (2010) afirma que los modelos educativos abarcan tres componentes, entre éstos se mencionan: un referente histórico, un sustento teórico y disciplinar y una concreción educativa. El mismo autor señala, además, que junto a esos tres elementos, es posible distinguir un conjunto de políticas públicas, las cuales sirven como elemento regulador para situar al modelo en concordancia con la perspectiva histórica.

Desde este enfoque, es asunción del investigador considerar que este autor ofrece una visión más integral de los elementos que debe contener un modelo educativo. Los mismos van desde el contexto histórico donde se desarrolle el modelo hasta los aspectos teóricos y educativos que encierra el mismo. A su vez, adiciona otros componentes que nutren la práctica educativa como lo son las tesis programáticas que le dan sustento a las teorías que orientan la implementación del modelo.

Finalmente, desde la posición de Valle Lima (2010), con quien el investigador coincide, un modelo educativo puede asumir como componentes los siguientes:

- Fin y objetivo

- Principios

- Caracterización del objeto de investigación

- Estrategia (Metodología, etc.)

- Formas de implementación

- Formas de evaluación

En este contexto, se presenta la figura 1 a objeto de representar las relaciones entre los componentes de un modelo educativo en concordancia a lo expresado por Valle Lima (2010): 


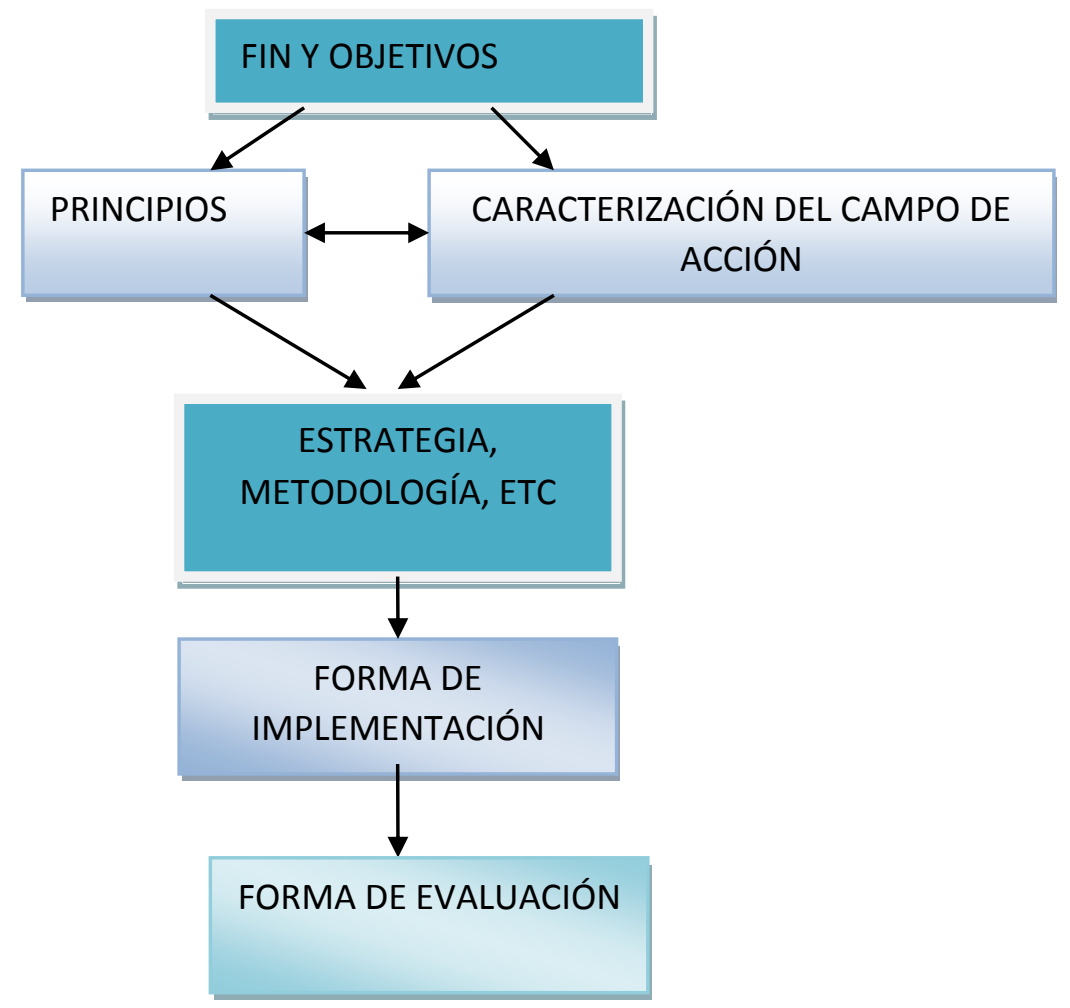

Figura $N^{\circ}$ 1. Componentes de un modelo educativo. Datos tomados de Valle Lima (2010).

Elaboración febrero 2019

En concordancia con el autor, desde el objeto de estudio se pueden abstraer el fin y objetivo, los principios y la caracterización del objeto de estudio. Así, el fin y objetivo describen lo que se quiere lograr con la puesta en práctica del modelo; los principios son las regularidades más generales que caracterizan al objeto de estudio y que guían su transformación; la caracterización del objeto de investigación, por su parte, es fundamental para llevar a cabo el proceso de valoración del modelo. Visto de este modo, la estrategia a implementar debe plantearse por etapas y cada una de éstas contiene objetivos, caracterización y las acciones a ejecutar. Las formas de implementación son los mecanismos y vías de instrumentación del modelo que comporta métodos, estrategias y la puesta en práctica del mismo y finalmente las formas de evaluación son las acciones tendentes a la valoración del desarrollo de la aplicación y resultados de la estrategia. 


\section{Esencialidades del modelo de investigación asumida}

El estudio sobre la gerencia estratégica para el fomento de la producción científica del profesor universitario, como categorías esenciales de esta investigación en ciencias sociales es comprendido desde el paradigma histórico-cultural según Toro y hurtado (2010), desde enfoque dialéctico-materialista. Siguiendo la posición de Delgado de Colmenares (2011:18), este tipo de investigación se concibe como un acto sustantivo que tiene el propósito de abordar las problemáticas y situaciones que afectan un contexto socioeducativo particular, con el fin de producir o descubrir nuevos elementos teóricos y realizar las acciones que modifiquen o transformen la realidad estudiada, a partir de recrear tanto lo cognitivo y valorativo como las prácticas cotidianas.

En este orden de ideas, Valle Lima (2010) sostiene que los problemas de la investigación científica pueden suscitarse en dos planos, a saber: el teórico y el práctico. En el primer orden, los problemas surgen en el marco de la teoría y contradicciones que no permiten encontrar una explicación adecuada a las mismas; mientras que en el segundo, los problemas se dan ante una situación que surge en la práctica cuando no hay una posible forma de dar solución a los mismos o cuando la solución que existe es para un problema similar, pero no ha sido probada en ese caso concreto. Es posición del autor de este estudio considerar, que los problemas sociales pueden emerger tanto del plano teórico como del plano práctico o de la unión entre ambos, tal es el caso de la investigación educativa, donde la solución de los mismos exige la unión dialéctica entre ambos planos.

En concordancia con lo señalado, Valle Lima (Op.Cit) refiere que todos estos resultados tanto teóricos como prácticos, en el campo de las ciencias de la educación se obtienen y se ponen en vigor en determinado marco social como parte de la formación del hombre que se quiere lograr. Por consiguiente, el resultado a obtener (tanto teórico como práctico) puede adquirir una connotación educativa, pedagógica o didáctica dependiendo el énfasis en el objetivo que se pretende lograr. Desde este marco, el investigador sustenta la investigación, ya que se hace hincapié en un resultado tanto teórico como práctico con una marcada connotación educativa, abordando el proceso para la formación del hombre, o de sus partes, en un plano social general, 
para fortalecer la gerencia estratégica para el fomento de la productividad científica del profesor universitario.

Una vez expuestas las consideraciones anteriores, el investigador asume como modo de abordar la realidad el enfoque dialéctico como marco de referencia teórico metodológico con sus respectivos supuestos ontológicos, gnoseológicos, lógicos y axiológicos, con la finalidad de describir, explicar y transformar la gerencia universitaria. Siguiendo a Castellano Simons (2005), el enfoque dialéctico entiende que la investigación educativa es un proceso orientado y regulado conscientemente, a lo largo de todos sus momentos y eslabones, por el método científico, como estrategia general del pensamiento científico que sigue el camino dialéctico de la cognición humana.

Así, a partir de la contemplación viva de los fenómenos educacionales, debe producirse el salto en el conocimiento hacia el pensamiento abstracto, para descubrir las esencias y relaciones con vistas a la modelación teórica del objeto, ascendiendo nuevamente a lo concreto pensado y regresando de nuevo a la práctica, lo que posibilitará solucionar los problemas inmediatos y perspectivos de la formación y desarrollo del ser humano.

Atendiendo a los componentes que deben integrar el modelo según el autor seguido, el investigador asume que un modelo educativo debe responder a las siguientes preguntas: ¿para qué educar?, ¿qué enseñar para lograrlo? y ¿cómo hacerlo de la manera más efectiva? A este respecto, el para qué debe contener en su definición del gerente que se requiere, los conocimientos y habilidades que debe poseer, tiene que ver, entre otros aspectos, con la centralidad del vínculo emocional entre vicerrectores, decanos, directores de programas, jefes de departamentos y de centros de investigación para que gestionen el proceso de docencia, investigación y extensión de manera integrada e integradora. De esta manera, a partir de los diferentes autores comentados, considerando sus contribuciones a esta investigación, se asumen los aportes de Valle Lima (2010) en relación con los elementos que deben conformar un modelo educativo. Se toman, además, como guía los aportes de Jara (2008) al considerar la importancia del establecimiento del marco filosófico, legal, organizativo adaptando los mismos al modelo a implementar y, finalmente, se comparten los elementos propuestos por Heredia (2016) considerándolos como ejes transversales en la elaboración del modelo. 


\section{Análisis y discusión de los resultados}

En este apartado se explican las esencialidades de la propuesta del modelo educativo para la gerencia estratégica que fomente la productividad científica del profesor universitario de la universidad Técnica de Manabí, sustentados en los aportes de Valle Lima (2010) y Jara (2008) referidos anteriormente. Los elementos que son los componentes del modelo que se propone, serían los siguientes:

- Fundamentos

- Principios

- Fin y Objetivo

- Caracterización de los actores sociales (Vicerrector, decano, directores de programa, jefes de departamentos, coordinadores de centros de investigación y profesores)

- Estrategia para el fortalecimiento de la producción científica del profesor universitario desde un modelo de gerencia estratégica.

- Formas de implementación

- Formas de evaluación

Asumido de este modo, los fundamentos y principios se constituyen en los componentes superiores del modelo educativo por ser regularidades generales de cumplimiento obligatorio y se encuentran en estrecha relación con el fin y objetivo que persigue el modelo. Se muestra, además, como componentes del modelo las formas de implementación y evaluación del mismo, las cuales están relacionadas con cada uno de los componentes que conforman el modelo educativo en la figura $\mathrm{N}^{\circ} 02$, de la siguiente guisa a saber: 


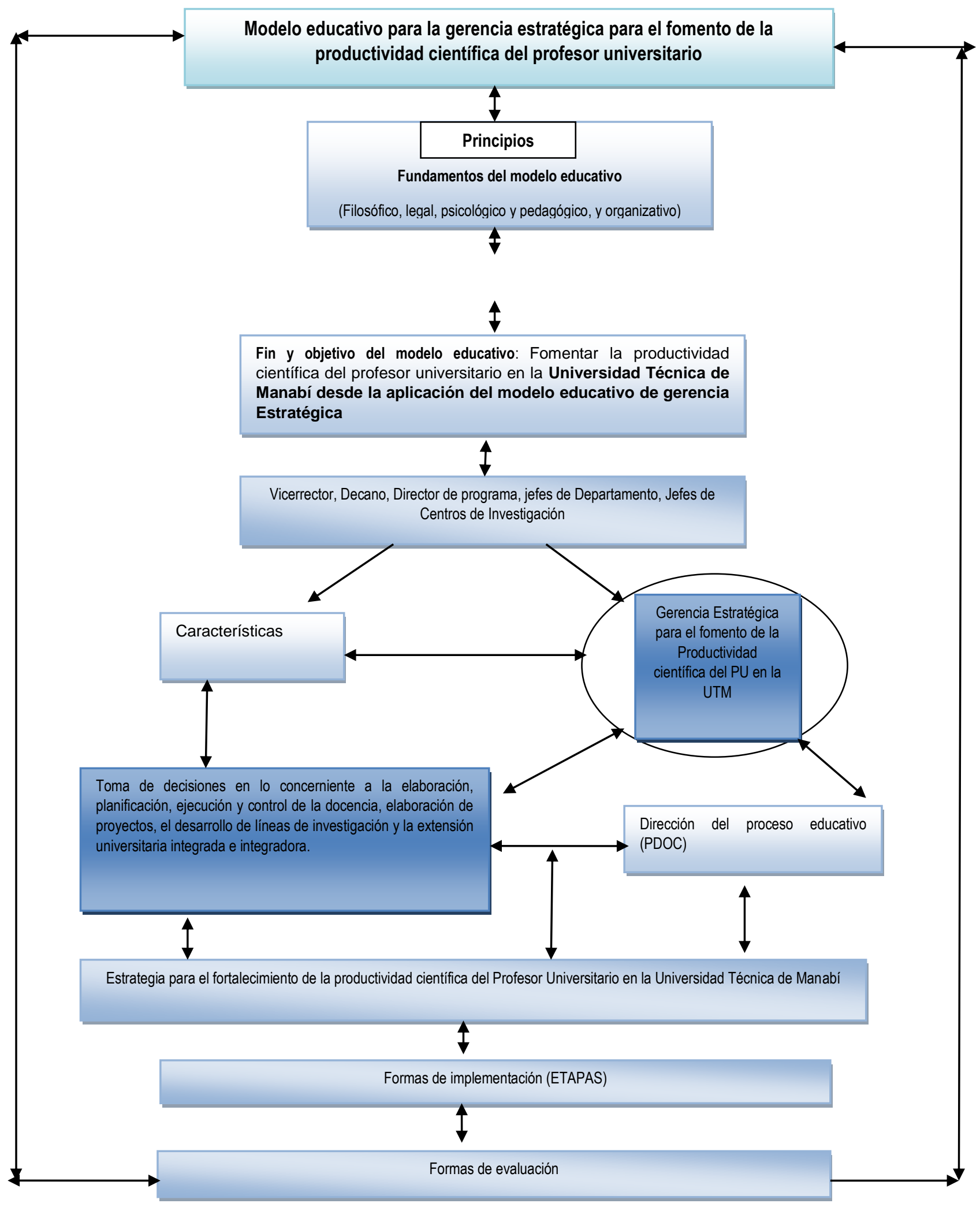


Figura Nª 02. Elementos del Modelo Educativo de Gerencia Estratégica para el fomento de la productividad Científica del profesor universitario. Fuente revisión bibliográfica de autores. Elaboración febrero 2019

\section{Fundamentos del modelo educativo}

\section{Filosóficos}

Este modelo educativo asume las posturas ontológicas, axiológicas, epistemológicas y sociológicas emanadas de la filosofía materialística dialéctica exaltando las capacidades de los profesores para integrar la docencia, la investigación y la extensión en orden de la toma de decisiones en todas las esferas del acontecer contextual en la que están inmersos. Por tanto, la modelación que se realice con los profesores universitarios debe ir encaminada hacia el enfrentamiento de todo el acontecer actual y participar en la toma de decisiones de su ámbito social, y por ende, que sea consecuente con las exigencias que demanda su entorno.

\section{Legales}

Este modelo se sustenta en la Sección quinta de la Constitución de Ecuador (2008) en correspondencia con el artículo 2, el 4, los artículos 6 y 8 en todos sus literales, el título I, el capítulo II sobre el personal académico de la Ley Orgánica de Educación Superior (2010).

\section{Psicológicos}

En la fundamentación psicológica del modelo se tomarán en cuenta los aportes realizados por la psicología marxista a través del enfoque histórico cultural creado por Lev Vigotsky (1979); así como también algunos elementos de la psicología humanista, entre los que destacan: el papel protagónico de quién está inserto en el proceso educativo, la importancia de la toma de decisiones y de la responsabilidad de los ciudadanos en sus procesos educativos sustantivos.

La vinculación entre los conceptos de educación y desarrollo desde la concepción vigotskiana fundamenta la dirección del proceso formativo de gerentes de la Universidad Técnica de Manabí es decir, la educación conduce al desarrollo pero debe tomarse en cuenta el desarrollo alcanzado y las potencialidades de cada uno de directores de los procesos universitarios sustantivos. De este 
modo, el concepto de Nivel de Desarrollo Actual es fundamento esencial del diagnóstico de las potencialidades y del desarrollo alcanzado por los profesores que ostentan los cargos de dirección de la universidad.

Otro aspecto de interés aportado por el enfoque histórico cultural al modelo que se propone es el de las ayudas (scaffolding) suficientes y necesarias que debe recibir cada persona por parte de sus pares en su actividad de organización. De importancia también resultan las contribuciones que desde la psicología humanista aportan algunos autores, al considerar el papel protagónico del ser, la importancia de la toma de decisiones y de la responsabilidad de las personas en su proceso directivo.

En lo concerniente a la categoría Gerencia Estratégica se asumen los supuestos propuestos por Sallenave (2002: p. 19) quien concibe al gerente como el "responsable de los ejes estratégicos de la empresa quien determina los objetivos y administra los recursos para lograrlos". Por su parte Kaplan y Norton (2005: p. 300) aportan que "las organizaciones basadas en la estrategia deben ser capaces de adaptarse a medida que el mundo cambia, de allí que sea menester que el enfoque de gerencia se sustente en lo estratégico en pos de mejorar y transformar la productividad científica de profesor universitario en la organización educativa en cuestión. Es así como se acepta lo referido por David (1999: p. 371), para quien la gerencia estratégica es aquella que "permite formar, ejecutar y evaluar acciones que conduzcan a la organización hacia el logro de sus objetivos."

Por su parte, Fonden (2005) refiere que el gerente debe desarrollar un plan estratégico en pos de fomentar la productividad científica del profesor universitario. En virtud de ello, se asumen los elementos de la gerencia estratégica como los componentes esenciales de la misma. Estos son la misión, la visión, orientadas hacia la planificación, organización, coordinación, dirección, control y evaluación de las actividades que fomentan la productividad científica del profesor universitario, tales como asistencia, organización y participación en eventos científicos, publicación de artículos, ponencias, libros, y otros que generen conocimientos.

Aunado a ello, en su tesis González (2014) afirma que el hecho gerencial implica asumir la responsabilidad de planificar, organizar, ejecutar, y controlar para dirigir la institución 
considerando para ello los propósitos de la organización, así como las estrategias para alcanzarlos en correspondencia con los planes y políticas educativas de la nación. Por ello la gerencia debe considerar los elementos de clima y cultura organizacional, tales como la misión, visión, valores, cultura y principios de la institución. (González, 2014: p. 20).

Asimismo este autor apunta que los gerentes deben ser profesionales que demuestren competencias profesionales que impulsen las transformaciones e innovaciones tecnológicas y alcance de objetivos en correspondencia con los valores de autonomía, responsabilidad y cooperación de la institución.

Por su parte, la productividad científica se entiende como la actividad que impulsa el logro de los resultados (productos de la docencia, de la investigación y de la extensión), optimizando los recursos en correspondencia con los valores y propósitos institucionales de la organización. Sin embargo, Fonden (2005) alerta lo que sigue a continuación: "Los equipos de trabajo pueden caer en improductividad y pasividad cuando no saben a dónde deben ir. Por consiguiente se precisa de una gerencia que establezca la dirección, los guíe con el ejemplo, los rete: esto no solamente los mantendrá en marcha sino que además hará sacar lo mejor de ellos".

La productividad científica es reflejo de calidad educativa y académica se corresponde con la relación recursos/ resultados obtenidos en cada una de las actividades que se desarrollan en la docencia (guías, separatas, compilaciones, dossier, planes y programas, unidades didácticas, diseño y creación de wikis, de aulas virtuales, diseño y creación de páginas web, de blog spot, elaboración de informes técnicos y académicos, planes de clase, diseños de power point con tecnología de punta, uso de las tics, aplicaciones pedagógicas de tecnología móvil, entre otras); en la investigación (tutoría y asesoría de proyectos de pre y postgraduación, publicación de investigaciones culminadas y artículos científicos, culminación y presentación de trabajos de ascenso, diseños de programas conducentes y no conducentes a grado (especializaciones, maestrías, diplomados, talleres, cursos de actualización, entre otros); publicación de capítulos, de libros, obtención de reconocimientos y premios académicos, participación y organización de eventos científicos como foros, simposios, congresos, jornadas y otros; en la extensión (difusión, 
conducción de programas de radio, atención, elaboración y ejecución de proyectos sociocomunitarios, proyectos socioproductivos, y otros).

En ese orden, se asumen los aportes de Cornejo (2011) afirma que el docente productivo es aquel que se distingue por su capacidad de cambio, que produce conocimientos y propiedad intelectual para el mundo académico y científico en el que se desenvuelve, alcanzando metas y objetivos al menor costo posible.

\section{Pedagógicos}

Basado, en primera instancia, en el modelo pedagógico social, el cual involucra el desarrollo de habilidades de pensamiento crítico-reflexivo que permitan al ciudadano y ciudadana participar activamente en los procesos de transformación de la sociedad se tomarán en cuenta las ideas sobre pensamiento crítico de Paulo Freire, Henry Giroux, Antonio Guiddens, Richard Paul y Linda Elder, entre otros.

Con relación a lo anterior, Freire (1987) afirma que se necesita un intercambio dialogal entre profesores y estudiantes, en la cual ambos aprenden, ambos cuestionan, ambos reflexionan y ambos participan en buscar significados. El investigador piensa que a través de este planteamiento, el autor deja ver la importancia de desarrollar el pensamiento crítico del ser humano con el fin de introducir mejoras en la sociedad y en el mundo en general. En líneas generales, el modelo se sustenta en los principios pedagógicos que abogan por una educación emancipadora y transformadora (Martín García y Barrientos Bradasic, (2009) para lograr un gerente estratégico que sea partícipe en la toma de decisiones, tanto en los proyectos a realizar en su entorno social como en todas las situaciones que exigen la participación activa y protagónica del mismo.

\section{Principios}

Los principios que rigen el modelo educativo aquí propuesto serán abordados desde la transversalidad, estos se mencionan a continuación: Interacción entre decanos, directores, jefes de departamento, coordinadores y profesores universitarios del Área de Ciencias de la Educación de la Universidad Técnica de Manabí 
a. Formación a cargo de un equipo multidisciplinario conformado por especialistas en áreas relacionadas con la psicología, sociología, educación, gerencia estratégica y productividad científica

b. Exaltación de una formación continua, basada en el desarrollo de habilidades de pensamiento crítico.

c. Orientación educativa socio-comunitaria y contextualizada a los sectores que circunscriben a la institución universitaria.

d. Dialogicidad permanente, intercambio de ideas y opiniones con criterio crítico entre los decanos, directores de programa, jefes de departamento y profesores.

e. Aplicación de técnicas de evaluación para comprobar la efectividad del modelo educativo.

\section{Objetivo General}

Fomentar la productividad científica de los profesores universitarios en la Universidad Técnica de Manabí, a objeto de introducir cambios en la toma de decisiones que conduzcan a la transformación cultural de la gestión universitaria.

\section{Objetivos específicos:}

- Sistematizar las concepciones teórico-metodológicas de la modelación de la gerencia estratégica para el fomento de la productividad científica de los profesores universitarios de la institución.

- Contribuir a la modelación de la gerencia estratégica para el fomento de la productividad científica de los profesores universitarios de la institución.

- Valorar el fortalecimiento de la gerencia estratégica para el fomento de la productividad científica de los profesores universitarios de la institución.

El gerente estratégico debe ser un Líder: debe ejercer influencia, atraer, movilizar y arrastrar con iniciativas y creatividad a quienes trabajan a su lado para que alcancen las metas que se han propuesto, así como conducir con éxito grupos formales e informales, propiciando la toma de conciencia, el compromiso y la unidad entre todos. Es posición del investigador pensar que los 
profesores que ostentan cargos directivos en las IES ameritan desarrollar habilidades para saber llevar a cabo todas sus tareas, por tanto, debe desarrollar su capacidad para saber hacer o ejecutar las acciones que le competen como director de la institución universitaria en orden a planificar, organizar, ejecutar y controlar la productividad científica del profesor.

\section{Conclusiones}

En la Universidad Técnica de Manabí se han observado las falencias existentes en cuanto a la productividad académica, por lo que es consideración del investigador pensar que se precisa la implementación de un modelo educativo de gerencia estratégica a tenor de fomentar la productividad científica en franca y directa relación con los tres procesos sustantivos de la función del docente universitario: docencia, investigación y extensión.

En ese sentido, se propone la implementación de un modelo educativo de gerencia estratégica para el fomento de la productividad académica sustentado en los postulados de Jara (2008), Valle (2010), conformado por objetivos, fundamentos psicológicos, pedagógicos, y gerenciales, en correspondencia con los aportes axiomáticos de autores como Sallenave (2002), David (1999), Fonden (2005), entre otros. A tenor de estimular, apoyar e impulsar el desarrollo de actividades científicas como Jornadas de investigación, congresos, simposios, foros en forma periódica, dado que son estos eventos los que en conjunto con otras acciones de docencia, investigación y extensión las que se configuran en fuente de producción de conocimientos y avances tecnológicos. 


\section{Referencias Bibliográficas}

Amorós, E. (2007). Comportamiento organizacional. USAT Escuela de Economía. Perú

Barreras, F. (2009). Modelo Pedagógico de Formación de Valores. Universidad Juan Marinello Vidaurreta. Matanzas, Cuba.

Bergues, M. (s.f). La modelación como método teórico de la investigación educativa. Recuperado en 17 de junio de 2019, de www.revistavarela.rimed.cu/articulos/rv2405.pd

Castellanos Simons, B.; Fernández González, A; Llivina Lavigne; Arencibia Sosa; Hernández Herrera, R. (2005). Esquema conceptual, referencial y operativo sobre la investigación educativa. Playa, Ciudad de La Habana: Editorial Pueblo y Educación

Centro de Asesoría Pedagógica (s.f). ¿Qué es un modelo? Recuperado en 5 de agosto de 2018, de http://es.catholic.net/op/articulos/42269/qu-es-un-modelo-educativo.html

Cerezal, J. y Fiallo, J. (2004). Cómo investigar en pedagogía. Ciudad de La Habana: Editorial Pueblo y Educación.

Chiavenato, I. (1999). Introducción a la Teoría General de la Administración. Colombia: Editorial McGraw-Hill.

Chirino, C. y Lamus, T. (2016) Modelo educativo para la participación comunitaria mediante el pensamiento crítico en la construcción de las comunas. Tesis en opción al Grado de Doctor en Ciencias para el Desarrollo Estratégico. UBV, Falcón, Venezuela.

Constitución de la República del Ecuador 2008. Decreto Legislativo 0. Registro Oficial 449 de 20-oct-2008. Última modificación: 13-jul-2011

Cornejo. M. (2011) Excelencia Directiva para lograr la productividad. Ebook Patagonia: México

David, F. (1999). La Gerencia Estratégica. Tercera Edición. Colombia: Editorial Legis. 
Delgado de Colmenares, F. (2011). Paradigmas y Retos de la Investigación Educativa. Una aproximación crítica. Mérida: Universidad de los Andes. Consejo de Publicaciones.

Farci, G. (2007). Patrones Metodológicos en la Evaluación de la Productividad y Producción Investigativa. Investigación y Postgrado- vol. 22 No. 1.

Foden, J. (2005). Áreas y tareas del director gerente. Sentido común para las 30 cuestiones que no puede ignorar. España: Deusto.

Franzoi, S. (2007). Psicología Social. México Mc Graw Hill.

Freire, P. (1987). Pedagogía del Oprimido (36a . Ed.). Montevideo: Siglo XXI Editores, S.A.

García, J. (2012). Cambio organizacional y disciplinario en unidades de investigación educativa. Plaza y Valdez. México DF.

García-Cepero, M. (2008). Panorama de las publicaciones seriadas y producción académica en el área de Educación, Iberoamérica. Magis, Revista Internacional de Investigación en Educación, 1(1),

Giroux, H. A. (1990). Los profesores como intelectuales. Hacia una pedagogía crítica del aprendizaje, Barcelona: Paidos.

González, C. (2006). Influencia de la Gerencia Estratégica en el Desempeño Laboral del Director de Educación Inicial. Maracaibo: URU (Trabajo especial de grado).

González, L. (2014). Nuevos roles del docente universitario en la sociedad del aprendizaje. Dale Carnegie Training. Bogotá. Colombia.

Guédez, V. (2006). Ética y práctica de la responsabilidad social empresarial. El aporte de la empresa al capital social. Caracas: Planeta.

Heredia, B. (2016). Modelo educativo ¿Qué es y por qué importa? Recuperado en 17 de junio de 2019, de http://www.elfinanciero.com.mx/opinion/modelo-educativo-que-es-y-por-queimporta.html 
Hurtado de Barrera, J. (2010). Cómo se elabora el proyecto de investigación. Tercera Edición, Fundación Sypal: Caracas. (Parte II Capítulo 3 y 4).

Ivancevich, J.; Konopaske, R., y Matteson, M. (2006). Comportamiento Organizacional. México: Mc Graw Hill.

Jara Vásquez, A. (2008) ¿Modelo educativo o modelo pedagógico? Deslinde conceptual entre modelo educativo y modelo pedagógico. Recuperado en 20 de agosto de 2018, de http://es.catholic.net/educadorescatolicos/694/2418/articulo.php?id=22081

Jiménez de Vargas, B. (1994). Productividad en investigación del docente universitario. Revista espacios. Vol. 14 (3). Caracas - Venezuela.

Jones, G., y George, J. (2006). Administración contemporánea. México: Mc Graw Hill

Kaplan, R., y Norton, D. (2005). Cómo utilizar el cuadro de mando integral para implantar y gestionar su estrategia. Barcelona (España: Gestión 2000)

Koontz, H. y Weihrich, H. (2012). Administración una perspectiva. México: Global.12a Edición

Koontz, H; Weihrich H; Cannice, M. (2008). Administración una perspectiva global y empresarial. México: Mc Graw Hill.

Ley Orgánica de Educación Superior, LOES. Registro Oficial Suplemento 298 de 12-oct-2010

López, A. (2008). Programas de estímulo a la productividad académica como expresión de una tendencia global. Revista Tribuna del Investigador Numero 2. Volumen 5

Martín García, A. Y Barrientos Bradasic, O. (2009). Los dominios del pensamiento crítico: una lectura desde la teoría de la educación. Universidad de Salamanca. Facultad de Educación. Departamento de Teoría e Historia de la Educación. Paseo de Canalejas, 169. 37008 Salamanca.

Martínez, H (2012) Seminario Internacional La Practica Pedagógica e entornos innovadores de aprendizaje. OEI Montevideo, Uruguay. 
Martínez, M. (2006) El paradigma emergente. Ediciones Trillas. Colombia México: Limusa Wiley.

Munévar, Dora Inés; Arana, Imelda y Agudelo, Catherín (2006). Productividad académica en la Universidad Nacional. Una aproximación crítica. Bogotá: Unibiblos.

Pérez Reynoso, Á. (2010). La intervención didáctica como alternativa para transformar la práctica. En: Revista de educación Nueva época, No 3, Octubre-Diciembre.

Pérez, A. (2009). Guía Metodológica para ante proyecto de Investigación. Caracas: FEDEUPEL

Perozo, S., de Arteaga, F. y Fuenmayor, B. (2008). La Productividad Investigativa de los Docentes del Instituto Universitario de Tecnología de Cabimas. Revista NEGOTIUM / Ciencias Gerenciales. Año 3 / Nº 9 / Abril.

Rodríguez, J. (2005). Decisiones Gerenciales Efectivas; Fundamentos para la solución de problemas administrativos. México: Trillas.

Sallenave, J-P (2002). Gerencia y Planeación Estratégica. Bogotá: Norma.

Schermerhom, J.; Hunt, J., y Osbom, R. (2005). Comportamiento Organizacional.

Schierenbeck (2010) Take on How to Bosst Productivity in Education. Springer Gabler.

Senlle, A. (2002). Señala. Calidad y liderazgo. Ediciones Gestión 2000, S.A.

Serna, H. (2000). Gerencia Estratégica. Planeación y gestión .Teoría y metodología. Santa Fe de Bogotá 3R Editores

UNESCO. (2003). Declaración mundial sobre la educación superior en el siglo XXI Visión y Acción. Paris.

Universidad Academia de Humanismo Cristiano. (2012). Vicerrectoría Académica - Unidad de Desarrollo de la Docencia. Recuperado en19 de septiembre de 2018, de http://www.geoacademia.cl/modeloeducativo.htm

Universidad de los Andes. (2012). Modelo educativo de la Universidad de los andes. Vicerrectorado académico. Consejo de desarrollo curricular. Mérida. Recuperado en 19 de 
septiembre de 2018, de ftp://tasa.arq.ula.ve/fadula/12.12.ModeloEducativo.ULA.CU-1-713.pdf

Valle Lima, A. (2010). La investigación pedagógica otra mirada. Ciudad de la Habana: Instituto central de ciencias pedagógicas.

Vega, G.; García, P.; Cartaya, H.; González, M. y Bonaci, S. (2011).Técnicas de decisión y sus procesos. Universidad nacional Experimental Simón Rodríguez. Recuperado en 30 de agosto de 2018, de http://wwwsimonrodriguez.blogspot.com/

Vigotski, L. (1979). El desarrollo de los procesos psicológicos superiores. Barcelona: Crítica Grijalbo 